\title{
Interpretación de la Práctica Docente en Educación Musical
}

${ }^{1}$ Centro Infantil Mi Carrusel, Colombia

julian.cardenas@javeriana.edu.co

2.Institucion Educativa Técnica López Quevedo

Como citar: Cárdenas Cárdenas, J., \& Fuentes Vargas, L. (2021). Interpretación de la Práctica Docente en Educación Musical. EDUCACIÓN Y CIENCLA, (24), e11777. https:// doi.org/10.19053/0120-7105. eyc.2020.24.e11777

\author{
Julián Andrés Cárdenas Cárdenas ${ }^{1}$ \\ Liseth Dayana Fuentes Vargas ${ }^{2}$
}

\section{Resumen}

El Conocimiento Didáctico del Contenido (CDC) es un concepto que explica cómo el maestro transforma su propio conocimiento en saber comprensible para los estudiantes. Para abordarlo, Park y Oliver proponen un modelo de seis componentes que interactúan en un contexto predefinido. Esta investigación busca hacer explícito el CDC de un profesor especialista en guitarra durante una clase de cuerdas pulsadas desarrollada en 2018, en un colegio privado de Tunja (Boyacá). Para ello, se hizo uso del modelo hexagonal propuesto por Park y Oliver a partir de un estudio de caso donde se analiza el planeamiento y grabación de una clase, así como, una entrevista al docente. Los resultados muestran que el CDC del educador se basa en el conocimiento práctico del instrumento y de los estudiantes, las representaciones y creencias sobre la clase y, la evaluación constante. De esta manera, en la materia se busca desarrollar la comprensión de los conceptos explicados mediante la ejecución instrumental que, a la vez, sirve como estrategia y medio de evaluación.

Palabras clave: Conocimiento Didáctico del Contenido, Didáctica, Educación Musical, Docente, Instrumentos de Cuerda.

Recibido: 22/09/2020 | Revisado: 10/12/2020

Aprobado: 15/12/2020 | Publicado: 31/12/2020 


\title{
Examining the Teacher Practice in Musical Education
}

\begin{abstract}
The Pedagogical Content Knowledge (PCK) is a concept that explains how the teacher transforms his own knowledge on the subject into understandable material for students. To address it, Park and Oliver propose a model of six components that interact in a predefined context. Aim of this research was to make explicit the PCK of a guitar specialist teacher during a plucked string class in 2018, at private school in Tunja (Boyacá). For this, the hexagonal model proposed by Park and Oliver was used based on a case study where the planning and recording of a class is analyzed, as well as an interview with the teacher. The results show that the teacher's PCK is based on the practical knowledge of the instrument and the students, the representations and beliefs about the class and, the constant evaluation. In this way, the subject proposes to develop the understanding of the explained concepts, through instrumental execution that, at the same time, serves as a strategy and way of evaluating.
\end{abstract}

Keywords: Pedagogical Content Knowledge, Pedagogy, Music Education, Music Teacher, Plucked Strings.

\section{Introducción}

El Conocimiento Didáctico del Contenido (CDC) es un concepto reportado originalmente por Lee Shulman (1986) que resalta la importancia de transformar el conocimiento propio de la materia en saber para la enseñanza. Así, el autor explica que existe una capacidad para adaptar aquello que los maestros entienden para sí mismos, en conceptos diseñados para el aprendizaje de los estudiantes, haciendo uso de metáforas, ejemplos demostrativos o nuevas formas de reorganización del saber (Shulman, 1987). El planteamiento inicial del CDC ha evolucionado hasta convertirse en una herramienta conceptual que sirve para comprender la manera en que funciona en la enseñanza y su proceso de desarrollo en diferentes disciplinas como las ciencias (Park \& Oliver, 2008), las matemáticas (Ball et al., 2008) y la música (Grieser \& Hendricks, 2018a; 2018b; Millican, 2013, 2016, 2017; Schiemann, 2017).

El abordaje teórico de este artículo plantea inicialmente una definición general de la didáctica, el CDC como concepto, la didáctica específica de la música, y finalmente, la conceptualización respecto al CDC del maestro de música. 


\section{Didáctica}

Actualmente, la didáctica puede ser definida como una disciplina científica que estudia la génesis, circulación y apropiación del saber y sus condiciones de enseñanza y aprendizaje (Zambrano, 2005). Este campo conceptual es reciente y surge como fruto de transformaciones sociales que tuvieron lugar a partir de 1960, así, resulta relevante el cambio de paradigma mediado por las tecnologías de la información y el uso transdisciplinar de las ciencias. Estos cambios terminaron por reorganizar las prácticas de producción económica y cultural repercutiendo en el sistema escolar (Zambrano, 2016). Se puede decir que, a partir de entonces, surge este nuevo concepto didáctico que redefine su estatus epistemológico y lo aleja del uso tradicional como atributo o adjetivo, para adentrarlo en el estudio y análisis de los fenómenos de enseñanza y aprendizaje de un contenido específico (Astolfi, 2001).

Bajo este último concepto, Astolfi (2001) encuentra que la didáctica está compuesta por tres elementos principales: alumno, saberes y enseñante, los cuales, están organizados en un triángulo donde las relaciones establecidas entre cada uno de sus vértices representan los sectores de interés. Estos nexos permitieron establecer los campos de investigación sobre la didáctica que dieron origen a conceptos como la ayuda didáctica, que resulta de la interacción entre enseñante y alumno

También, la identificación del objeto de estudio y de los actores principales de la didáctica favorecieron el surgimiento del interés por delimitar y profundizar en campos más específicos de conocimiento, los cuales, fueron denominados posteriormente como didácticas específicas. Esta delimitación se concentra en cinco criterios que articulan las dinámicas sociales a las que genera respuesta la didáctica, estos son: los distintos niveles de formación del sistema educativo, las edades de los alumnos, el campo disciplinar y las subdivisiones específicas que tienen lugar al interior de la didáctica, el tipo de institución y su tipo de formación (formal o no formal), por último, las características de los sujetos (Camilloni et al., 2007). En este contexto teórico surge la didáctica de la música, teniendo en cuenta las particularidades de las familias de instrumentos.

\section{Conocimiento Didáctico del Contenido}

Uno de los campos de interés investigativo que surgen a partir del establecimiento de la didáctica como disciplina científica, se da en torno a la inquietud por comprender de manera profunda la naturaleza de la relación entre el enseñante, su saber y contribución en la enseñanza. El CDC es un concepto desarrollado por Shulman (1986) y su equipo de investigación, como marco teórico para la explicación del fenómeno donde se transforma el conocimiento disciplinar que posee el maestro, en representaciones didácticas para la enseñanza. Este cambio supuso que el ámbito investigativo considerara el conocimiento para la enseñanza como un tipo de saber práctico y personal propio del docente (Bolívar, 2005). 
Por ello, el CDC permitió cambiar la forma en que se investigaba al maestro y su conocimiento disciplinar. Inicialmente, las investigaciones intentaron vincular estadísticamente el saber específico de los maestros con los aprendizajes de los estudiantes, sin embargo, las nuevas dimensiones aportadas por el CDC permitieron entender que el conocimiento del docente sobre la materia impartida "abarca más de lo que es típicamente medido en test estandarizados de elección múltiple y el número de clases que alguien ha tomado” (Grossman et al., 2005, p.6).

A partir de entonces, estudios recientes se centraron en indagar sobre el problema del conocimiento en relación con la enseñanza, encontrando que los docentes "adaptan su conocimiento del contenido al contexto en el que están enseñando" (Grossman et al., 2005, p.6), esto les permite desarrollar un saber específico diferente al que posee un científico, ya que, incluyen una contextualización psicológica de la disciplina para poder enseñarla. En este sentido, es posible reconocer el CDC como resultado de la experiencia del educador, donde interactúan el conocimiento de la materia y la reflexión en la práctica para favorecer la comprensión de un tema o campo específico de conocimiento. El CDC es, por lo tanto, el conocimiento práctico que le da forma a la profesión del maestro.

\section{Conocimiento Didáctico del Contenido en Música}

Pese a que el CDC ha sido un campo de investigación prolífico, es importante aclarar que el cuerpo de estudios respecto a la didáctica de la música es todavía muy incipiente (Conway, 1999; Grieser \& Hendricks, 2018a; Millican, 2013). De hecho, es apropiado afirmar que el estudio mismo de la didáctica de la música se encuentra en formación y por este motivo, aún es frecuente encontrar ejemplos donde el término didáctica se confunde fácilmente con otros campos y conceptos como currículo, metodologías o lúdica (Martínez, 2016).

No obstante, para el campo musical Conway (1999) menciona que el CDC es entendido como un conocimiento que incluye la comprensión de los problemas asociados con el aprendizaje de un instrumento musical y las estrategias necesarias para obtener una enseñanza musical instrumental exitosa. El autor también agrega que este conocimiento puede provenir del currículo y los objetivos, organización de los contenidos de la clase, reclutamiento e instrumentación balanceada, organización de los ensayos, elección de la literatura apropiada, manejo de la clase en ensayos, motivación, evaluación y calificación, musicalidad, y, relación o comprensión de los estudiantes.

A su vez, Haston y Leon-Guerrero (2008) resaltan que los trabajos realizados con el propósito de comprender el CDC de maestros de música, permiten determinar como eje de desarrollo la apropiación de componentes kinestésicos y estéticos en los estudiantes. Por otro lado, Ballantyne y Packer (2004) definen el CDC en música como el conocimiento de las técnicas de educación musical que favorece vínculos 
significativos del estudiante con la música, la implementación del currículo de manera efectiva, el cálculo de las distintas habilidades musicales de los estudiantes, y, la explicación y demostración de conceptos musicales.

\section{Conocimiento Didáctico del Contenido. Revisión Elaborada por Park y Oliver (2008)}

Continuando con el gran interés investigativo que sobrevino tras el desarrollo del concepto del CDC, Soonhye Park y Steve Oliver (2008) reexaminaron este constructo para desarrollar una herramienta conceptual que facilitara la comunicación entre los investigadores educativos, los formadores de maestros y los docentes, para, principalmente, evaluar el CDC una vez construido. Aunque la investigación se realizó con profesores de ciencias sus aportes pueden ser trasladados para comprender el funcionamiento del CDC en otras áreas de formación como la música (Rojas, 2019).

Los hallazgos de Park y Oliver (2008) pueden agruparse en dos bloques, por un lado, los autores identifican la eficiencia del maestro como un componente crucial en el desarrollo del CDC. Por otro lado, deducen que el CDC resulta influenciado por las preguntas de los estudiantes, su pensamiento crítico, respuestas verbales y no verbales y la evidencia de su aprendizaje. Además, aclaran que, la comprensión que desarrollan los profesores respecto de los mal entendidos que tienen los estudiantes, resultan un factor clave en el desarrollo del CDC, pues esto ocurre como resultado de la reflexión docente sobre la importancia del rol del estudiante en el desarrollo del CDC.

A partir de estos hallazgos, Park y Oliver (2008) sugieren representar el concepto CDC mediante un esquema hexagonal que relaciona las orientaciones para la enseñanza, el conocimiento sobre la comprensión de los estudiantes, el conocimiento del currículo, el conocimiento de las estrategias y representaciones para la enseñanza, el conocimiento de la evaluación y la eficacia del profesor, con el contexto. Es pertinente aclarar que esta integración es posible gracias a la reflexión en y sobre la acción que realiza el maestro.

Ahora bien, existen otros aportes pertinentes para la comprensión del CDC que, aunque no se encuentran organizados bajo la lógica del modelo de Park y Oliver (2008), pueden resultar útiles para el presente estudio. Por este motivo, a continuación, se condensa y clasifica la información de cada componente

\section{Componentes del Modelo Hexagonal de Park \& Oliver y Didáctica Específica de la Música}

Orientaciones para la Enseñanza de la Música. Bajo este componente se agrupan las creencias de los docentes respecto de los objetivos, propósitos y naturaleza de la enseñanza, lo cual, a su vez, le sirve al docente como hoja de ruta conceptual (Park \& Oliver, 2008). Para la educación musical, ese componente se 
hizo explícito a través de distintos métodos que a lo largo del siglo XX abordaron la enseñanza del ritmo, la melodía, la armonía y el dominio instrumental (Hemsy de Gainza, 2003); elementos que pueden ser desarrollados de manera individual o vinculados directamente con el aprendizaje de un instrumento musical.

Dentro de la didáctica de los instrumentos musicales, además se ha reconocido la existencia de dos componentes básicos que interactúan durante el proceso de interpretación musical, por un lado, el componente técnico, donde se encuentran los elementos operativos que hacen posible la activación del instrumento musical, $\mathrm{y}$, por otro lado, el componente expresivo, que agrupa y organiza los factores bajo los cuales el músico puede tomar las decisiones interpretativas (Sloboda, 2000). Desde una perspectiva específica, para la didáctica de la guitarra se puede reconocer la posición de la guitarra y el ejecutante, las funciones de las manos derecha e izquierda, la independencia entre las manos, el control físico y mental del sonido $\mathrm{y}$, el fraseo en la ejecución de golpes, ritmos y melodías, como objetivos de los componentes técnico y expresivo (Glise, 1997; Vásquez, 2017).

El Conocimiento de las Estrategias y Representaciones para la Enseñanza de la Música Según la definición aportada por Park y Oliver (2008), este componente organiza el CDC del docente en dos categorías relacionadas con las estrategias para la enseñanza, en este caso, de la música. En primer lugar, se encuentran las que son propias de la asignatura, de carácter general y se relacionan con los objetivos que cada maestro concibe. En segundo lugar, aparecen las habilidades propias del tema, subdivididas, a su vez, en actividades y representaciones diseñadas para la enseñanza de aspectos específicos contenidos dentro del dominio disciplinar.

En este sentido, Schiemann (2017) desarrolla un estudio donde inicialmente pretende determinar las secuencias o estrategias utilizadas por docentes especialistas y generalistas, y la inclusión de apoyo verbal y no verbal en la práctica musical durante una clase de música en primaria. El estudio determinó que los profesores generalistas tendían a utilizar menos tiempo de la clase apoyando a los estudiantes con instrumentos musicales temperados y una mayor cantidad del tiempo cantando y reproduciendo ritmos. En contraste, los maestros especialistas invertían más tiempo interpretando y apoyando a los estudiantes durante la ejecución de los instrumentos musicales. Estas estrategias utilizadas por los profesores especialistas, ocurren comúnmente dentro de las clases individuales de instrumento donde existe una relación maestro-aprendiz más estrecha (Young et al., 2003).

Otro estudio realizado por Haston y Leon-Guerrero (2008), encontró que la construcción del CDC en el componente de estrategias y representaciones, se desarrollaba en mayor medida, desde la experiencia y aprendizaje instrumental de los maestros principiantes, pues gran parte de las actividades realizadas en clase provenían de lo que habían visto en otros docentes, de las estrategias que habían sido utilizadas por sus profesores de instrumento y de su experiencia en 
el trabajo colectivo de ensambles. Sin embargo, es necesario aclarar que, para la enseñanza de instrumentos de cuerda donde pueden existir diversos niveles de dominio en habilidades motrices o expresivas, e inclusive distintos tipos de instrumentos interactuando de manera simultánea, es necesario que el profesor incluya conocimiento didáctico y de la materia, para seleccionar y adaptar las estrategias apropiadas (Grieser \& Hendricks, 2018b).

Conocimiento de la Comprensión de los Estudiantes. Park y Oliver (2008) subrayan que, para que un maestro pueda utilizar de manera efectiva el CDC es importante que comprenda cuáles son los conocimientos previos que tienen sus estudiantes frente a un tema determinado, reconozca la dificultad que para ellos representa, distinga la motivación, pertinencia y necesidad del tema e indague sobre los estilos de aprendizaje en los que se movilizan sus estudiantes.

En lo que concierne a la didáctica de la música, es posible señalar que diversos estudios realizados por Si Millican, han demostrado que la comprensión de los estudiantes es un componente crucial en el desarrollo del CDC para los docentes de música. De esta manera, mientras que los estudiantes de educación musical se confunden al momento de identificar y diferenciar los problemas de interpretación y sus posibles causas (Millican, 2016), aquellos maestros más experimentados, teniendo como base su experiencia interpretativa, articulan las soluciones necesarias para atender problemas relacionados a la ejecución e interpretación instrumental, así mismo, estos profesores han desarrollado formas eficientes para conocer e identificar de manera conjunta con sus aprendices las dificultades que presentan (Millican, 2013). En adición a los aspectos anteriores, se identificó que los educadores experimentados desarrollan un tipo de lenguaje que les permite modelar aquello que quieren enseñar desde términos significativos para los estudiantes (Millican, 2017), lo que demuestra la comprensión de las representaciones que tienen los educandos respecto a diferentes temas.

Por otro lado, el conocimiento que tienen los profesores sobre las dificultades que pueden presentar algunos estudiantes en el proceso de aprendizaje musical, les ayuda a una planeación de clase efectiva, a la vez que, los orienta para seleccionar las herramientas de apoyo adecuadas tanto al interior de clases de educación vocal (Freer, 2018), como instrumentales (Zingara, 2004).

Conocimiento del Currículo. Este aspecto le permite al docente tener un campo de enunciación desde un marco conceptual y legal (Park \& Oliver, 2008). Siguiendo a Park y Oliver (2008) este tipo de conocimiento proviene del material curricular (flexible y no flexible) desde donde el maestro toma referencia para elegir los contenidos, actividades y conceptos básicos, desde una postura que integra los lineamientos curriculares de la materia con los factores que condicionan la enseñanza y el aprendizaje. 
En Colombia, en términos curriculares, la música como asignatura se integra al sistema educativo nacional en el área de educación artística que articula artes visuales, música, teatro y danza (MEN, 2010). Sin embargo, durante el IV Encuentro de Educación Artística celebrado por el Ministerio de Educación Nacional (MEN) en Bogotá (2008) se determinó que la formación en esta área debe favorecer una educación por las artes, es decir, el arte para una formación de valores. Partiendo de estas premisas se entiende al arte como un campo de saber y expresión que desarrolla tres competencias específicas: sensibilidad, apreciación estética y comunicación, las cuales, a su vez, se desprenden de procesos de recepción (percepción-análisis), creación (apropiación-creación) y socialización (presentación pública-gestión), en el marco de una dimensión social, cultural y subjetiva (MEN, 2010).

Por otro lado, el MEN considera al área de educación artística como transversal en el currículo de formación, desde donde se vincula directamente a las competencias básicas. No obstante, las instituciones educativas tienen la potestad de seleccionar el contenido de la línea a orientar (música, teatro, danza, artes visuales) dependiendo de sus intereses, recursos y necesidades (MEN, 2010). Sin embargo, a la fecha el MEN no ha designado derechos básicos de aprendizaje en educación artística, razón por la cual, es posible afirmar que el docente tiene libertad de contenido bajo los parámetros de la institución educativa en la que labora.

Conocimiento de la Evaluación. En este componente se vinculan el conocimiento de las dimensiones más relevantes para la evaluación y los métodos que sirven para evaluar cada dimensión apropiadamente.

De acuerdo al MEN existen tres dimensiones en la educación artística que son susceptibles de ser evaluadas, estas son, las habilidades técnicas, los aspectos estéticos y expresivos y la imaginación creadora. Las primeras sirven "para dar forma física a una idea o a una intuición” (MEN, 2010 p. 75). Por otro lado, los aspectos estéticos y expresivos se refieren a la capacidad que posee el estudiante de manifestar y representar físicamente su apreciación y expresión sensible, para que, a través de su argumentación el docente comprenda "cuándo el estudiante afronta un problema técnico o un problema estético, cuándo se le dificulta o facilita comprender las relaciones conceptuales y hasta dónde un docente debe mediar o intervenir” (MEN, 2010 p. 75). Por último, la imaginación creadora precisamente se relaciona con la habilidad del estudiante para hacer visible el uso de la imaginación en sus productos, este es un aspecto que, aunque depende de la habilidad técnica no se relaciona directamente con ella, por lo tanto, tiene diversos grados de exigencia como la ampliación de límites, la invención, la ruptura de límites y la organización estética.

De otra parte, la valoración en educación artística se puede efectuar desde diversas perspectivas, como lo es la evaluación diagnostica que le permite al docente comprender los conocimientos e intereses de sus estudiantes. La evaluación sujeta 
al criterio personalizado, donde el diálogo estudiante-profesor permite seguir y valorar los procesos individuales de la clase. La coevaluación posibilita que los estudiantes puedan socializar sus productos y emitan un juicio crítico sobre el trabajo individual y del otro. Así mismo, la autoevaluación, entendida como la búsqueda que hace el docente por generar autocrítica en sus estudiantes, y por último, la indagación, donde el docente puede dar cuenta de procesos reflexivos sobre su práctica (MEN, 2010).

Eficacia del profesor. Park y Oliver (2008) encuentran esta categoría como resultado de situaciones específicas del aula. Este campo también ha sido objeto de algunas investigaciones referidas al CDC en la música. Dentro de los estudios, se destaca los llevados a cabo por Millican (2013) donde encontró que el CDC provenía de la interacción de distintas áreas, destacando en primer lugar, el papel esencial del dominio instrumental, puesto que durante las etapas iniciales de formación de un intérprete se requiere de un buen referente auditivo, y en segundo lugar, es primordial que el maestro tenga una representación mental clara de aquello que quiere oír en sus alumnos. Hallazgos similares fueron develados por Schiemann (2017) que resalta la importancia de los ejemplos provistos por el profesor durante las etapas formativas.

Como resulta evidente hasta este punto, aunque los componentes del modelo hexagonal diseñado por Park y Oliver (2008) fueron concebidos inicialmente para el estudio del CDC de docentes de ciencias, sus categorías de indagación permiten organizar un marco teórico apto para el análisis de otras áreas del conocimiento como la música. En tanto, y tomando como referencia la conceptualización propuesta, se analizará el CDC de un profesor especialista de música durante una clase de guitarra en la ciudad de Tunja (Boyacá).

\section{Metodología}

\section{Método}

La presente investigación enmarcada dentro del paradigma cualitativo, utilizó como herramienta metodológica el estudio de caso pues este permite comprender y abarcar la complejidad de un caso particular, sin la búsqueda de generalización (Stake, 1999). Se seleccionó el estudio de caso instrumental, teniendo en cuenta que ayuda a condensar los datos para conocer y comprender profundamente un fenómeno, que en este caso se relaciona con la forma en que interactúan los componentes de la CDC del profesor durante la clase de guitarra (Stake, 1999).

\section{Participante}

En este estudio participó voluntariamente un docente especialista en guitarra, egresado en el 2016 del programa de formación musical que ofrece el Servicio Nacional de Aprendizaje. El maestro cuenta con experiencia profesional de dos 
años y medio como docente de música en un colegio de carácter privado de la ciudad de Tunja, donde hace parte del programa de formación artística no formal. Así mismo, es un intérprete activo de bajo eléctrico y guitarra en diferentes agrupaciones de música comercial.

Para este estudio se realizó una selección no probabilística por conveniencia, orientada a satisfacer los objetivos expuestos (Hernández et al., 2014).

\section{Técnicas de Recolección}

Para la recolección de la información se utilizó el planeador de clase del profesor, la grabación de una clase de guitarra y una entrevista semiestructurada que buscó profundizar en la comprensión de lo sucedido. El protocolo de la entrevista fue diseñado teniendo en cuenta los componentes que integran el esquema hexagonal diseñado por Park y Oliver (2008), adaptado a las características de la didáctica de la música. Así mismo, fue evaluado por dos pares investigadores que aportaron sugerencias incluidas en el protocolo final.

Como método para validar la investigación, se empleó la triangulación de las fuentes de datos para así contrastar la información desde espacios y puntos de vista distintos (Stake, 1999). También se abordó la triangulación de investigadores, como estrategia para validar la fiabilidad cualitativa y la credibilidad, al comparar y analizar los datos desde diferentes perspectivas (Hernández et al., 2014).

\section{Procedimiento}

Recolección de la Información. Tras la firma del consentimiento informado donde se comunicó y consensuó con el colegio y el maestro la investigación, se procedió a solicitar copia del planeador de clases del Docente Participante (DP).

A continuación, se realizó la grabación del audio de una clase (dirigida a estudiantes de tercero, cuarto y quinto primaria) donde el DP fue el único profesor presente, pues se buscó no interferir de manera directa sobre el ambiente de aprendizaje del aula. La grabación [G] tiene una extensión de 50 minutos, posteriormente se realizó la entrevista [E] con el DP. La clase aborda, en palabras del docente:

enseñanza de una parte de la canción Zombie (The Cranberries, 1994) en guitarra a los niños de tercero, cuarto y quinto, como preparativo para la muestra del programa de formación artística, que será realizado en el mes de octubre $[G]$

Análisis de los datos. Se empleó el software Atlas Ti (octava versión) como herramienta para examinar la información bajo las orientaciones propias de la teoría fundamentada (Strauss \& Corbin, 2002). Así, el proceso inició con la codificación abierta que buscó conceptualizar y clasificar los acontecimientos, actos y resultados de las tres fuentes de información, a través, de un microanálisis que permitió 
organizar los datos y códigos para los que se tuvieron en cuenta características, propiedades o dimensiones similares. Luego, se realizó una codificación axial u organización jerárquica de los códigos en categorías. Por último, se dio paso a una codificación selectiva que integra las categorías jerárquicamente superiores dentro de un esquema general (Strauss \& Corbin, 2002).

\section{Resultados}

Los resultados se organizaron de acuerdo a cada uno de los ejes temáticos que conforman el concepto de CDC del DP y sus manifestaciones.

\section{Orientaciones para la enseñanza de la música}

En torno a este componente se destaca que el DP reconoce el dominio del instrumento y de los elementos básicos de la música (armonía o acordes, ritmo y melodía) como ejes fundamentales en la práctica.

Debe ser enseñado el conocimiento del instrumento, logrando un manejo adecuado del mismo, para que sea interpretado funcionalmente. Para mi es necesario que los muchachos tengan un conocimiento de elementos fundamentales de la música, porque al lograr que los estudiantes dominen los elementos básicos de la música, pueden acercarse a la parte artística que ellos tienen y de esta manera adquirir mayores destrezas [E].

Tal parece que el profesor trabajó la comprensión de estos conceptos a partir de la práctica con el instrumento. Así, por ejemplo, demostró la melodía y procedió a introducir el concepto de síncopa explicando el trabajo individual de las manos para la interpretación de la síncopa en guitarra.

\section{Conocimiento de la Comprensión de los Estudiantes}

Es posible afirmar que este componente es una de las tres categorías (junto con el conocimiento de las estrategias y representaciones para la enseñanza de la música, y las orientaciones para la enseñanza de la música) más relevantes para el docente. En su concepto, es muy importante que los estudiantes participen activamente realizando preguntas, porque así, puede resolver dudas de manera personalizada ejecutando con el instrumento los conceptos abordados en clase, pues permite que los discentes confronten su representación sobre lo aprendido teóricamente en partituras (teoría musical) con el componente práctico de interpretación instrumental. Al respecto el docente menciona:

La teoría musical en algunos aspectos llega a ser difícil de hacer entender, pero en la mayoría de los casos, gracias a la guitarra se puede trabajar [E].

Otro aspecto a resaltar en torno a la comprensión de los estudiantes, se relaciona con la preocupación del DP por incorporar aspectos que motiven a participar en su clase. Aunque el maestro es consciente que la participación de los estudiantes es 
voluntaria, ya que el colegio ofrece cuatro líneas de formación artística en donde la clase de música puede ser elegida por los estudiantes, el profesor permite que sean los aprendices quienes decidan qué canción quieren interpretar, en este caso Zombie, The Cranberies, para tomarla como eje temático desde donde planea su clase. Al respecto indica que:

Al comienzo elegimos entre todos qué canciones vamos a estudiar en clase y dependiendo de eso, yo elaboro los arreglos y el material para trabajar las canciones [E].

Lo anterior puede guardar relación con el hecho que la institución educativa sugiere a sus profesores organizar y planear las clases desde las necesidades de los estudiantes.

Siempre nos recomiendan hacer un sondeo general de los estudiantes y ver muy bien sus capacidades y aptitudes para poder realizar un seguimiento individual de los estudiantes y plantear objetivos sólidos [E].

\section{Conocimiento del Currículo}

Este es uno de los componentes más ausentes en los resultados de la investigación, aunque se encontró que el docente desarrolló contenidos durante la clase soportado en el plan de aula y de área, el maestro manifestó que no conoce ni se apoya en ningún documento institucional de orden nacional como las Orientaciones pedagógicas para la educación artística en básica y media (MEN, 2010) o los Lineamientos curriculares de educación artística (MEN, 2000). Sin embargo, el planeador de clase permite evidenciar que los tres componentes que reconoce el MEN (2000) como esenciales (sensibilidad, apreciación estética y comunicación) tienen espacio en la clase. Por ejemplo, a lo largo del año se llevan a cabo muestras públicas grupales y sesiones de reconocimiento y apreciación.

\section{Conocimiento de la Evaluación}

Si bien la clase de guitarra es una asignatura de libre elección, esta influye en el promedio académico del estudiante, por lo tanto, necesita ser evaluada. Para este fin, el docente hace uso de su conocimiento de la materia y realiza la evaluación teniendo en cuenta los ejes temáticos que orientan su enseñanza. Al respecto menciona:

Considero importante evaluar el trabajo en clase y el desarrollo de ejercicios de gramática y solfeo que se desarrollan en grupo talleres, trabajos en grupo, desarrollo de tareas [E].

Aunque no fue señalado explícitamente, el desarrollo de la clase permite ver que se hace un seguimiento constante y casi personalizado a la ejecución instrumental que, como se mencionó anteriormente, congrega el conocimiento teórico (gramática, solfeo, armonía) dentro un producto sonoro perceptible. 


\section{Conocimiento de las Estrategias y Representaciones para la Enseñanza de la Música}

En este apartado, también se hizo evidente la preferencia por la ejecución instrumental como estrategia para transformar el contenido en una representación adecuada para los estudiantes. Por ello, cada explicación de un tema o fragmento estuvo seguido de una demostración con el instrumento, tanto en las actividades colectivas, como en las individuales:

A ver niños, ahora vamos a hacer el acompañamiento de la primera parte del coro así (profesor demuestra el fragmento en cuestión con la guitarra) pongan mucha atención al ritmo en la mano derecha, vamos a hacerlo así (demostrando con la guitarra) dos abajo, uno arriba, dos abajo, uno arriba, uno arriba, uno abajo, con el ritmo que está en el tablero ¿listos? Vamos a ver todos, uno, dos, tres, cuatro (los niños y el profesor inician a tocar) [G].

Por otro lado, la metodología de trabajo fue diseñada basándose principalmente en la experiencia del profesor, quien manifiesta

El 70\% del material de la clase es elaborado por mí y también uso algunos métodos que expliquen mejor el uso de la guitarra, de lectura rítmica y solfeo [E].

\section{Eficacia del Profesor}

En torno a este componente, se encontró que el conocimiento y dominio del instrumento musical es contemplado como elemento clave al momento de enseñar. A la pregunta ¿Qué habilidades o destrezas propias considera más pertinentes para el desarrollo de la clase? El profesor respondió:

El conocimiento y manejo de varios instrumentos [E].

Este hecho se hizo evidente durante el desarrollo de la clase, pues, como ya se mencionó, la estrategia de enseñanza más utilizada fue la ejemplificación de los temas a través de la interpretación instrumental.

\section{Conclusiones}

Tras los resultados de esta investigación se hace evidente que la construcción del CDC del docente surge como resultado de la interacción de los componentes que integran el esquema hexagonal diseñado por Park y Oliver (2008) mediante la reflexión en y sobre la práctica.

A través de la reflexión sobre su práctica, el DP adopta estrategias para la comprensión de los estudiantes como la ejemplificación de los temas abordados por medio de la interpretación de la guitarra y el diseño del material de enseñanza basado en su experiencia de aprendizaje, permitiendo demostrar que, como reconoce Hemsy de Gainza (2003), las orientaciones para la enseñanza de la música son el resultado de la unión entre los conceptos de armonía, melodía y ritmo, con 
elementos clave de la interpretación de la guitarra. Así, en concordancia con los hallazgos de Millican (2013) y Schiemann (2017) las habilidades interpretativas del profesor son reconocidas como una de las características fundamentales para la enseñanza de la música, porque respaldan las orientaciones para la enseñanza del docente, su eficacia, y las estrategias y representaciones presentes en la clase.

Así mismo, la permanente reflexión del DP le permite comprender y evaluar el desempeño de los estudiantes, con el objetivo de adaptar las estrategias y en algunos casos, el contenido de clase. De esta manera, además de preguntar constantemente con el ánimo de monitorear la comprensión de los estudiantes, elemento que coincide con los resultados de Millican (2013), el profesor elige de manera conjunta con sus estudiantes el tema a abordar en clase para, partiendo de ello, crear el material que permita el proceso de aprendizaje, teniendo en cuenta, las capacidades musicales con las que empiezan el año los estudiantes. Igualmente, el DP demuestra un saber implícito al realizar una constante evaluación diagnóstica buscando reconocer la comprensión del contenido de la clase, aunque desconozca las recomendaciones en lineamientos curriculares para la educación artística.

Es posible señalar que el CDC del DP se cimienta en su conocimiento práctico del instrumento, su capacidad para estar en constante diálogo con los estudiantes y comprenderlos, su saber implícito de los mecanismos evaluativos adecuados, las orientaciones comunes para la enseñanza musical (ritmo, melodía, armonía y dominio instrumental) y el desarrollo de estrategias propias para la clase. Sin embargo, carece de conocimiento curricular explícito, por lo tanto, algunos componentes básicos como la imaginación creativa queda por fuera de su conocimiento y su hacer.

\section{Referencias Bibliográficas}

Astolfi, J. P. (2001). Conceptos clave en la didáctica de las disciplinas. Díada.

Ball, D. L., Thames, M. H., \& Phelps, G. (2008). Content knowledge for teaching: What makes it special. Journal of teacher education, 59(5), 389-407. https://doi. org/10.1177/0022487108324554

Ballantyne, J., \& Packer, J. (2004). Effectiveness of preservice music teacher education programs: Perceptions of early-career music teachers. Music Education Research, 6(3), 299-312. https://doi.org/10.1080/1461380042000281749

Bolívar, A. (2005). Conocimiento didáctico del contenido y didácticas específicas. Profesorado. Revista de currículum y formación del profesorado, 9(2), 1-39.

Camilloni, A., Cols, E., Basabe, L., \& Fenney, S. (2007). El Saber Didáctico. Paidós.

Conway, C. M. (1999). The development of teaching cases for instrumental music methods courses. Journal of Research in Music Education, 47(4), 343-356. https:// doi.org/10.2307/3345489 
Freer, P. K. (2018). Research-to-Resource: Initial Steps in Vocal Technique for Boys Experiencing Difficulty With Phonation During the Adolescent Voice Change. Update: Applications of Research in Music Education, 37(1), 9-12. https:// doi.org/10.1177/8755123318779880

Hemsy de Gainza, V. (2003). La educación musical entre dos siglos: del modelo metodológico a los nuevos paradigmas. (Documento de trabajo $\mathrm{N}^{\circ} 10$ ). Universidad de San Andrés. http//hdl.handle.met/10908/773

Glise, A. L. (1997). Classical guitarpedagogy. a handbook for teachers. Mel Bay Publications.

Grieser, D., \& Hendricks, K. (2018a). Pedagogical Content Knowledge for Shifting: More than a Toolbox of Tricks. American String Teacher, 68(2), 16-19. https://doi. org/10.1177/0003131318760822

Grieser, D., \& Hendricks, K. (2018b). Review of Literature: Pedagogical Content Knowledge and String Teacher Preparation. Update: Applications of Research in Music Education, 37(1), 13-19. https://doi.org/10.1177/8755123318760970

Grossman, L. P., Wilson, S. M., \& Shulman, L. S. (2005). Profesores de sustancia: el conocimiento de la materia para la enseñanza (P. V. Rodríguez, Trad.). Profesorado, Revista de currículum y formación delprofesorado, 9(2), 1-25. (Trabajo original publicado en 1989) https://recyt.fecyt.es/index.php/profesorado/article/view/42676

Haston, W., \& Leon-Guerrero, A. (2008). Sources of Pedagogical Content Knowledge Reports by Preservice Instrumental Music Teacher. Journal of Music Teacher Education 17(2), 48-59. https://doi.org/10.1177/1057083708317644

Hernández, R., Fernández, C., \& Baptista M. (2014). Metodología de la investigación (6a ed.). McGraw-Hill

Martínez, G. (2016). Estado del arte de la didáctica de la música desde una revisión de la revista Eufonía en los años 2014, 2015 [Trabajo de grado de especialización, Universidad Pedagógica Nacional]. https://goo.gl/YTUdPE

Millican, J. S. (2013). Describing Instrumental Music Teachers' Thinking: Implications for Understanding Pedagogical Content Knowledge. Update: Applications Of Research In Music Education, 31(2), 45-53. https://doi. org/10.1177/8755123312473761

Millican, J. S. (2016). Describing Preservice Instrumental Music Educators' Pedagogical Content Knowledge. Update: Applications of Research in Music Education, 34(2), 61-68. https://doi.org/10.1177/8755123314552664

Millican, J. S. (2017). Examining Pedagogical Content Knowledge of an Expert Band Director Teaching Lips Slurs. Journal of Music Teacher Education, 26(2), 90 103. https://doi.org/10.1177/1057083716629610 
Ministerio de Educación Nacional. (2010). Orientaciones pedagógicas para la educación artística en básica y Media. (Documento $\mathrm{N}^{\circ}$ 16). https://www.mineducacion.gov. co/1759/articles-241907_archivo_pdf_orientaciones_artes.pdf

Ministerio de Educación Nacional. (2008). IV encuentro Nacional de Educación Artística: Lugares y sentidos del arte en la educación en Colombia (Relatorías finales). MEN.

Ministerio de Educación Nacional. (2000). Lineamientos curriculares de educación artística (Serie de lineamientos curriculares, educación artística) https://www. mineducacion.gov.co/1621/articles-339975_recurso_4.pdf

Park, S., \& Oliver, J. S. (2008). Revisiting the conceptualization of pedagogical content knowledge (PCK): PCK as a conceptual tool to understand teachers as professionals. Research in Science Education, 38(3), 261-284. https://doi.org/10.1007/s11165-009-9163-8

Rojas, G. A. (2019). Conocimiento didáctico del contenido como técnica interpretativa de una práctica docente musical. Estudios Artísticos, 5(7), 209-225. https://doi.org/10.14483/25009311.14989

Schiemann, S. (2017). Generalist and specialist primary music teachers' uses of nonverbal and verbal support during music practice. Psychomusicology: Music, Mind, and Brain, 27(3), 180-190. https://doi.org/10.1037/pmu0000189

Shulman, L. S. (1986). Those Who Understand: Knowledge Growth in Teaching. Educational Researcher, 15(2), 4-14. https://doi.org/10.3102/0013189X015002004

Shulman, L. S. (1987). Knowledge and Teaching: Foundations of the New Reform. Harvard Educational Review, 57(1), 1-23. https://doi.org/10.17763/ haer.57.1.j463w79r56455411

Sloboda, J. A. (2000). Individual Differences in Music Performance. Trends in Cognitive Sciences, 4(10), 397-403. https://doi.org/10.1016/S1364-6613(00)01531-X

Stake, R. E. (1999). Investigación con Estudio de Casos. Morata

Strauss, A., \& Corbin, J. (2002). Bases de la investigación cualitativa. Técnicas y procedimientos para desarrollar la teoría fundamentada (E. Zimmerman, Trad.; 1a ed.). Universidad de Antioquia.

Vásquez, C. M. (2017). Entre Cuerdas de Guitarra. PNMC. https://cutt.ly/sdR7hk6

Young, V., Burwell, K., \& Pickup, D. (2003). Areas of Study and Teaching Strategies in Instrumental Teaching: a case study research project. Music Education Research, 5(2), 139-155. https://doi.org/10.1080/14613800307110

Zambrano, A. (2005). Didáctica, pedagogía y saber. Cooperativa Editorial Magisterio.

Zambrano, A. (2016). Pedagogía y didáctica: esbozo de las diferencias, tensiones y relaciones de dos campos. Praxis \& Saber, 7(13), 45-61. https://doi. org/10.19053/22160159.4159 
Zingara, J. J. (2004). Cleaning up Trumpet Sound: Some Paths to Better Tone. Teaching Music, 12(3), 42. 